\title{
Management of severe hidradenitis suppurativa with biologic therapy and wide excision
}

\author{
Shi Yu Derek Lim ${ }^{1}$, Ee Cherk Cheong ${ }^{2}$, Hazel H. Oon ${ }^{3}$ \\ ${ }^{I}$ National Healthcare Group, Singapore; ${ }^{2}$ Plastic, Reconstructive and Aesthetic Surgery Service, Department of General Surgery, Tan Tock \\ Seng Hospital, Singapore; ${ }^{3}$ National Skin Centre, Singapore
}

Hidradenitis suppurativa (HS) is a chronic inflammatory follicular occlusive disease that involves the intertriginous areas. Treatment methods include conventional topical and systemic medication, radiotherapy, biologic agents, and surgical excision. Of late, there has been an increased focus on the use of biologic agents in patients with moderate to severe HS. Here, we present the case of a 46-year-old man with Hurley stage III HS for whom wide excision was ultimately curative, after aggressive medical therapy with the use of infliximab and adalimumab had succeeded in limiting the body surface area affected by the disease. This case demonstrates the effective treatment of severe HS with a combination of biologic therapy and surgery.

Keywords Hidradenitis suppurativa / Tumor necrosis factor-alpha / Antibodies, monoclonal / Adalimumab / Infliximab
Correspondence: Shi Yu Derek Lim National Healthcare Group, c/o Hazel Oon, National Skin Centre,

1 Mandalay Road, Singapore 308205, Singapore

Tel: $+65-62534455$

Fax: $+65-62533225$

E-mail:dereklim@ymail.com

Received: 23 Mar $2018 \bullet$ Revised: 23 Oct $2018 \bullet$ Accepted: 30 Oct 2018

pISSN: 2234-6163・ elSSN: 2234-6171 • https://doi.org/10.5999/aps.2018.00339・Arch Plast Surg 2019;46:272-276

\section{INTRODUCTION}

Hidradenitis suppurativa (HS), also known as acne inversa, is a chronic inflammatory follicular occlusive disease affecting the intertriginous folds. The manifestations of this disease can vary from inflammatory nodules and abscesses to the formation of sinus tracts and scarring [1]. Treatment is challenging, and in severe cases, surgery is required for control, in combination with medical management [2]. In view of the challenges in the treatment of HS, several biologic agents have been used as targeted therapy for the disease. To date, adalimumab is the only Food and Drug Administration (FDA)-approved biologic agent for the treatment of HS, but several other biologic therapies have shown great promise. We present a case of an Asian man with severe HS for whom wide excision with staged split-thickness skin grafting was ultimately curative, after aggressive medical therapy with the use of biologics had succeeded in limiting the body surface area affected by the disease.

\section{CASE}

A 46-year-old man first presented with Hurley stage III HS affecting the intertriginous areas for more than 20 years. He gave a history of an incision and drainage procedure on his gluteal region 28 years ago prior and had self-medicated with traditional Chinese medication. On examination, multiple tender nodules and overlying abscesses, together with double-headed comedones and contracted scars were noted affecting his axillae, groins, and buttocks.

He was treated with isotretinoin, doxycycline, erythromycin, ciprofloxacin, and combination therapy with oral clindamycin and rifampicin with minimal efficacy. Subsequently, he was 
placed on a combination of acitretin, metformin, and intravenous infliximab infusions, of which he received 17 doses over 18 months. On this regimen, the body surface area affected by HS initially improved greatly, decreasing from the initial area of involvement (bilateral axillae, groin, and buttocks) to involve only an approximately $25-\mathrm{cm}$-wide area of indurated plaques and nodules on the left buttock (Fig. 1). He was offered surgery but repeatedly declined, as he was fearful of the morbidity associated with the procedure. He also declined radiotherapy. His disease showed minimal sustained improvement despite switching from infliximab to regular subcutaneous adalimumab injections for 9 months. In total, he underwent 27 months of biologic therapy, with an initial excellent response, but persistent left buttock disease. His Dermatology Life Quality Index (DLQI) score was 18 out of 30, indicating a very large impact on his quality of life. The DLQI is a self-administered questionnaire used to measure the impact of a patient's skin disease on quality of life [3].

The patient then finally consented to surgery. Magnetic resonance imaging and ultrasound of the remaining affected left gluteal region showed a complex intersphincteric fistulous network. He underwent wide excision of the left gluteal lesion with fistulotomies and application of continuous high-intensity negative-pressure dressing at $-125 \mathrm{mmHg}$ on the left gluteal defect (Fig. 2). The intervening application of negative-pressure dressing, before eventual skin grafting at a later date, served two purposes. It prepared the bed with granulation tissue for the subsequent skin grafts, and also allowed further inspection of the surgical field at a later date to confirm that the bed was indeed free of residual HS and contained no anal fistula, meaning that it was suitable for skin grafting. A defunctioning sigmoid colostomy was also performed during wide excision surgery to manage his complex anal fistula and to prevent fecal soiling of his gluteal wound, which was in close proximity to the anal region. A splitthickness skin graft was performed seven days later with a negative-pressure dressing sponge applied over a layer of lipido-colloid polyester mesh dressings on the skin grafts to secure them to the wound bed, without adhering to them. The negativepressure dressing was removed 6 days later, and the skin grafts were noted to be well-taken. Medical therapy for HS was stopped after surgery. Two months after skin grafting, the left gluteal region was observed to be well-healed and disease-free (Fig. 3). However, he developed a small anal fistula beyond the area of excision and skin graft site, for which he underwent fistulotomy. He has been followed up for a year thereafter with no recurrence of disease.

The patient provided consent for his photographs to be taken for publication.

\section{Fig. 2. Left gluteal region (intraoperative)}

Figure shows the left gluteal region after excision of the left gluteal lesion (intraoperative view).

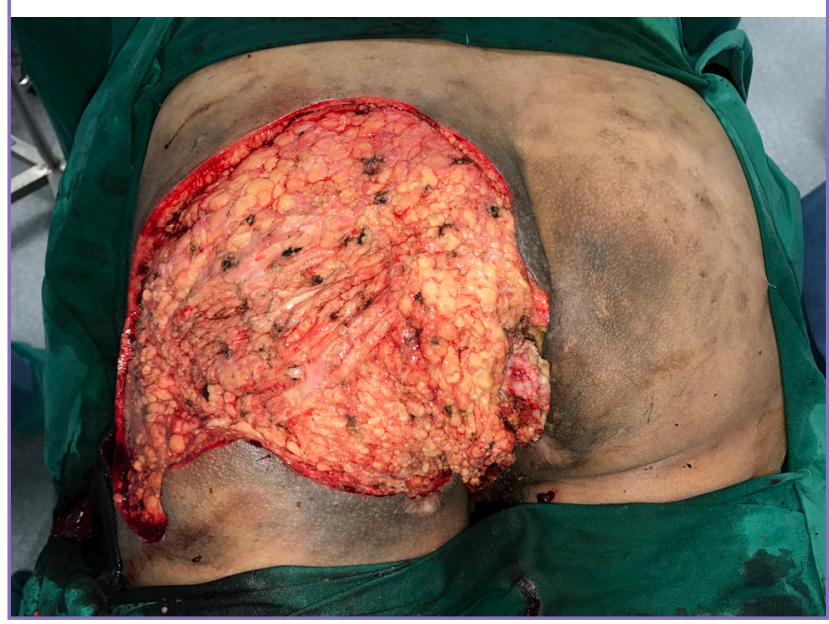

Fig. 1. Suprapubic, inguinal and left gluteal regions (preoperative)

(A) Figure shows contracted scars, with no active lesions, over the inguinal and suprapubic region. (B) Figure shows indurated plaques and nodules over the left gluteal region despite biologic therapy with 17 rounds of intravenous infliximab and 9 months of adalimumab.
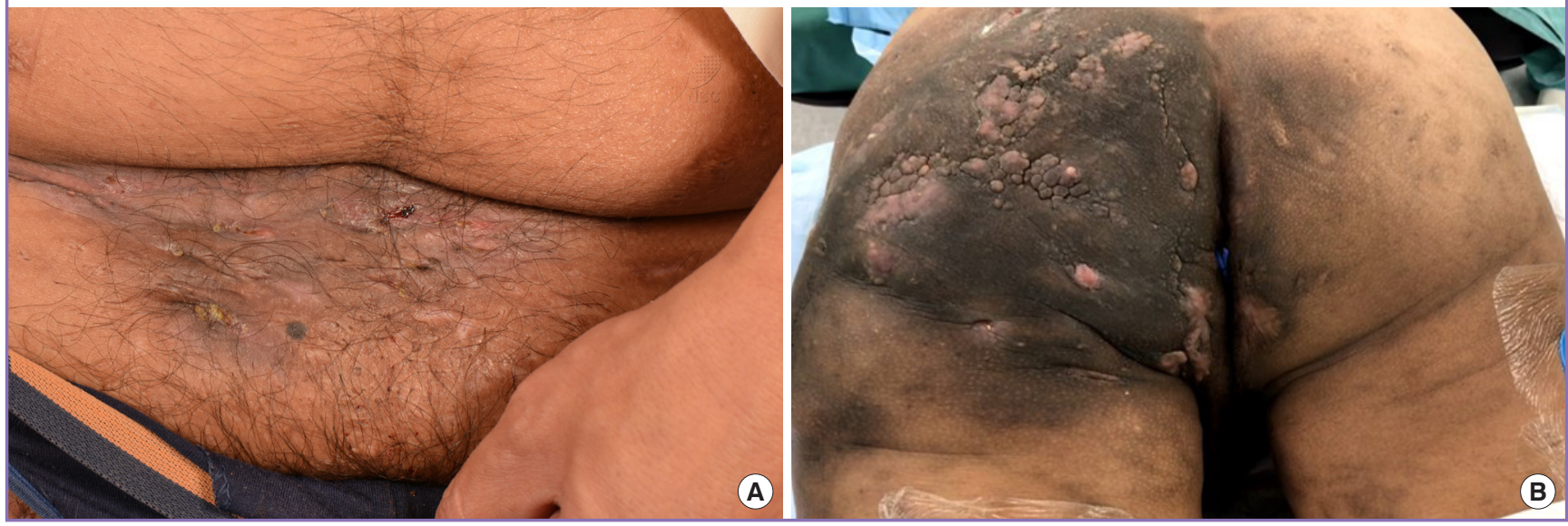


\section{DISCUSSION}

A treatment algorithm for $\mathrm{HS}$ was proposed in 2016 by Gulliver et al. [4] (Table 1). In addition, other biologic therapies, as elaborated below, should be considered in cases of moderate to severe $\mathrm{HS}$ in the event of standard regimen failure.

Adalimumab, a recombinant human anti-IgG1 tumor necrosis factor-alpha (TNF- $\alpha$ ) monoclonal antibody, was FDA-approved for the treatment of moderate/severe HS in 2015, and remains the only FDA-approved biologic agent to date. It is given subcutaneously at an initial dose of $160 \mathrm{mg}$, followed by $80 \mathrm{mg} 2$ weeks later and a maintenance dose of $40 \mathrm{mg}$ weekly thereafter. Based on the PIONEER I and PIONEER II trials, the weekly administration of $40 \mathrm{mg}$ of adalimumab in patients with moderate/severe $\mathrm{HS}$ resulted in significantly higher clinical response rates and the achievement of HS clinical response (HiSCR) af-

\section{Fig. 3. Left gluteal region ( 2 months post-skin grafting)}

Figure shows the well-healed, disease-free left gluteal region 2 months after split-thickness skin grafting.

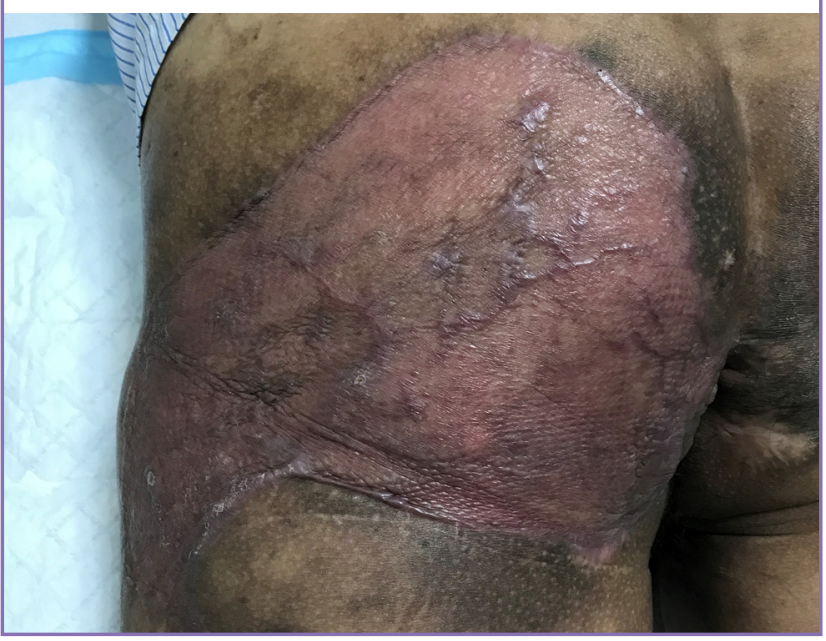

ter 12 weeks, defined as a 50\% reduction in abscess and inflammatory nodule counts, with no increase in abscess or draining fistula counts [5]. The majority of adverse events observed during the PIONEER I and II trials were mild or moderate in severity. Of note, there were rare reports of new psoriasiform eruptions and psoriasis, one case of nasal squamous cell carcinoma, and one death from cardiorespiratory arrest 42 days after the last dose of adalimumab in a 35-year-old man with a history of diabetes mellitus and smoking and a family history of ischemic heart disease [5]. An open-label extension trial of the PIONEER I and II trials up to 168 weeks also demonstrated longterm response in patients on adalimumab with no new safety risks [6].

An increasing number of other biologic therapies are being trialled in the treatment of moderate/severe HS refractory to conventional medical treatment. These clinical trials have shown promising results.

Infliximab, a chimeric mouse/human anti-TNF- $\alpha$ monoclonal antibody, has been used as an off-label treatment in patients with HS resistant to adalimumab. It is dosed as an intravenous infusion of $5 \mathrm{mg} / \mathrm{kg}$ body weight at weeks 0,2 , and 6 and thereafter every 8 weeks. In a phase II trial involving 38 patients with moderate to severe HS, more patients treated with infliximab showed a greater than $25 \%$ reduction in the HS Severity Index compared to patients treated with placebo [7]. Most adverse events described were mild, such as influenza-like illnesses and a case of herpes simplex infection. There was one case of infusion reaction resulting in hospitalization [7].

In our patient, infliximab was given initially as there was no FDA-approved biologic at the initiation of treatment in 2013. This decision was made based on then-published evidence from phase II trials of adalimumab and infliximab, although a direct comparison between the clinical trials was challenging, as they

Table 1. Summary of treatment algorithm for hidradenitis suppurativa

\begin{tabular}{|c|c|c|c|}
\hline Severity of HS & First-line treatment & Second-line treatment & Third-line treatment \\
\hline Hurley stage I & $\begin{array}{l}\text { Topical clindamycin lotion 1\% } \\
\text { Oral tetracycline }\end{array}$ & $\begin{array}{l}\text { Topical resorcinol } \\
\text { Zinc gluconate } \\
\text { Intralesional corticosteroids } \\
\text { Acitretin } \\
\text { Etretinate }\end{array}$ & $\begin{array}{l}\text { Colchicine } \\
\text { Botulinum toxin } \\
\text { Isotretinoin } \\
\text { Dapsone } \\
\text { Cyclosporine } \\
\text { Hormones }\end{array}$ \\
\hline Hurley stage II & $\begin{array}{l}\text { Treatment as for stage I HS } \\
\text { Oral clindamycin with rifampicin } \\
\text { Subcutaneous adalimumab (if unresponsive or intolerant to oral antibiotics) }\end{array}$ & $\begin{array}{l}\text { Treatment as for stage I HS } \\
\text { Intravenous infliximab }\end{array}$ & Treatment as for stage I HS \\
\hline Hurley stage III & Treatment as for stage I and II HS & & \\
\hline All & $\begin{array}{l}\text { Need for surgical intervention to be assessed in all patients based on type } \\
\text { and extent of scarring }\end{array}$ & & \\
\hline
\end{tabular}


utilized different severity scoring systems and clinical endpoints $[7,8]$. Subsequently, infliximab was switched to adalimumab, as the continued effect from infliximab had diminished and the patient still had significant persistent residual disease.

Ustekinumab is a subcutaneously administered human monoclonal antibody that acts by binding to the $\mathrm{p} 40$ subunit on interleukin (IL)-12 and IL-23. In a phase II open-label study involving 17 patients on ustekinumab, the majority of patients showed moderate-to-marked improvement, as defined by a significant decrease in the modified Sartorius Scale and modified HS Lesional Area Severity Index. Forty-seven percent of the patients achieved HiSCR [9]. The dosing regimen for ustekinumab in HS requires further refinement, and the authors of the phase II study suggested the need for increased dosage for greater efficacy. All adverse events described were mild and temporary [9].

Anakinra, a recombinant IL-1 receptor A antagonist, has been studied for the treatment of HS. In a double-blind, randomized, placebo-controlled trial involving 20 patients with moderate to severe HS, 78\% of patients given anakinra daily achieved HiS$\mathrm{CR}$, compared to only $30 \%$ of patients given placebo $(\mathrm{P}=0.04)$ after 12 weeks of treatment. However, there was no significant difference seen at week 24 [10]. Painful injection site reactions are commonly reported with anakinra, which may limit its tolerability for patients [11].

Canakinumab, a human monoclonal anti-IL-1 $\beta$ antibody, has shown mixed results in case reports [12,13]. Other biologic therapies under investigation in phase $\mathrm{I} / \mathrm{II}$ trials include MABp1 (an anti-IL-1 $\alpha$ human monoclonal antibody), secukinumab (an anti-IL-17A human antibody), and IFX-1 (an anti-C5a monoclonal antibody) [14]. One of the difficulties in directly comparing the outcomes of these trials lies in the varying clinical measurement scores and endpoints used to determine effectiveness of treatment.

With the rise of biologic therapy, it is important to emphasize that prior to initiation, patients should undergo screening for latent infections such as tuberculosis, human immunodeficiency virus, and hepatitis $\mathrm{B}$ and $\mathrm{C}$, as immunosuppression can reactivate and exacerbate these conditions [15].

Severe HS can be extremely challenging to treat. It is frustrating for doctors and patients alike when there is a lack of sustained response to treatment. This case highlights the need to combine aggressive medical therapy with surgical excision to achieve disease remission and cure. Effective medical therapy can assist in debulking the inflammatory disease burden prior to surgical therapy. Surgery eliminates draining fistulae and scars, as well as reduces the chance of subsequent recurrence. As more biologic agents become available for HS, a multidisciplinary approach to treatment, involving a plastic surgeon and a dermatol- ogist familiar with the use of biologics, as well as allied healthcare staff trained in the holistic management of these patients, is of paramount importance. Patients who have shown a plateaued response to medical treatment should be considered for early surgical intervention in view of the potential for drastic improvement.

\section{NOTES}

\section{Conflict of interest}

HHO is a clinical investigator for Janssen, Novartis, and Pfizer. She has also served as a speaker and advisory board member for AbbVie, Janssen, Novartis, and Eli Lilly. No other potential conflict of interest relevant to this article was reported.

\section{Ethical approval}

The study was performed in accordance with the principles of the Declaration of Helsinki. Written informed consent was obtained.

\section{Patient consent}

The patient provided written informed consent for the publication and the use of his images.

\section{Author contribution}

Writing - original draft: Lim SY. Writing - review and editing: Cheong EC. Conceptualization, writing - review and editing: Oon HH. Approval of final manuscript: all authors.

\section{ORCID}

Shi Yu Derek Lim https://orcid.org/0000-0002-7079-2416

Ee Cherk Cheong https://orcid.org/0000-0002-4897-4029

Hazel H. Oon https://orcid.org/0000-0001-7607-3124

\section{REFERENCES}

1. Sellheyer K, Krahl D. "Hidradenitis suppurativa" is acne inversa! An appeal to (finally) abandon a misnomer. Int J Dermatol 2005; 44:535-40.

2. Zouboulis CC, Desai N, Emtestam L, et al. European S1 guideline for the treatment of hidradenitis suppurativa/acne inversa. J Eur Acad Dermatol Venereol 2015;29:619-44.

3. Finlay AY, Khan GK. Dermatology Life Quality Index (DLQI): a simple practical measure for routine clinical use. Clin Exp Dermatol 1994;19:210-6.

4. Gulliver W, Zouboulis CC, Prens E, et al. Evidence-based approach to the treatment of hidradenitis suppurativa/acne inversa, based on the European guidelines for hidradenitis 
suppurativa. Rev Endocr Metab Disord 2016;17:343-51.

5. Kimball AB, Okun MM, Williams DA, et al. Two phase 3 trials of adalimumab for hidradenitis suppurativa. $\mathrm{N}$ Engl J Med 2016;375:422-34.

6. Zouboulis CC, Okun MM, Prens EP, et al. Long-term adalimumab efficacy in patients with moderate-to-severe hidradenitis suppurativa/acne inversa: 3-year results of a phase 3 open-label extension study. J Am Acad Dermatol 2019;80: 60-9.

7. Grant A, Gonzalez T, Montgomery MO, et al. Infliximab therapy for patients with moderate to severe hidradenitis suppurativa: a randomized, double-blind, placebo-controlled crossover trial. J Am Acad Dermatol 2010;62:20517.

8. Kimball AB, Sobell JM, Zouboulis CC, et al. HiSCR (Hidradenitis Suppurativa Clinical Response): a novel clinical endpoint to evaluate therapeutic outcomes in patients with hidradenitis suppurativa from the placebo-controlled portion of a phase 2 adalimumab study. J Eur Acad Dermatol Venereol 2016;30:989-94.

9. Blok JL, Li K, Brodmerkel C, et al. Ustekinumab in hidradenitis suppurativa: clinical results and a search for potential biomarkers in serum. Br J Dermatol 2016;174:839-46.
10. Tzanetakou V, Kanni T, Giatrakou S, et al. Safety and efficacy of anakinra in severe hidradenitis suppurativa: a randomized clinical trial. JAMA Dermatol 2016;152:52-9.

11. Leslie KS, Tripathi SV, Nguyen TV, et al. An open-label study of anakinra for the treatment of moderate to severe hidradenitis suppurativa. J Am Acad Dermatol 2014;70:24351.

12. Houriet C, Seyed Jafari SM, Thomi R, et al. Canakinumab for severe hidradenitis suppurativa: preliminary experience in 2 cases. JAMA Dermatol 2017;153:1195-7.

13. Sun NZ, Ro T, Jolly P, et al. Non-response to interleukin-1 antagonist canakinumab in two patients with refractory pyoderma gangrenosum and hidradenitis suppurativa. J Clin Aesthet Dermatol 2017;10:36-8.

14. Theut Riis P, Thorlacius LR, Jemec GB. Investigational drugs in clinical trials for hidradenitis suppurativa. Expert Opin Investig Drugs 2018;27:43-53.

15. Ahn CS, Dothard EH, Garner ML, et al. To test or not to test? An updated evidence-based assessment of the value of screening and monitoring tests when using systemic biologic agents to treat psoriasis and psoriatic arthritis. J Am Acad Dermatol 2015;73:420-8. 